Pacific Journal of Mathematics

ON BISIMPLE WEAKLY INVERSE SEMIGROUPS 


\title{
ON BISIMPLE WEAKLY INVERSE SEMIGROUPS
}

\section{S. MADHAVAN}

\begin{abstract}
A regular semigroup $S$ with a commutative subsemigroup of idempotents $E$ is called weakly inverse if for any $a \in S$ the set $E_{a}$ of inverses $a^{\prime}$ of $a$ for which $a^{\prime} a \in E$ is nonempty and for all, $a, b \in S, E_{a b} \subseteq E_{b} E_{a}$ and $E_{a}=E_{b} \Rightarrow a=b$. In this paper we show that in a weakly inverse semigroup $S$ with partial identities the $\mathscr{R}$-class $R$ which contains the partial identities is a right skew semigroup and conversely, every right skew semigroup $R$ may be so represented. If $R$ satisfies the condition that for every $a, b \in R$ there exists a $c \in R$ such that $R a \cap R b=R c$, then our considerations lead to a construction of bisimple weakly inverse semigroup with partial identities.
\end{abstract}

The weakly inverse semigroups have been introduced and investigated by B. R. Srinivasan [5] and the results we have obtained generalize same results of Reilly [4] concerning bisimple inverse semigroups.

2. Preliminaries. We assume that the reader is familiar with some of the basic results of [2].

Let $S$ be a semigroup. An idempotent $e$ of $S$ is called a principal idempotent of $S$ if $f e f=f e$ for every idempotent $f$ of $S$ An element $a$ of $S$ is called a principal element of $S$ if there exists an inverse $a^{\prime}$ of $S$ such that $a a^{\prime}$ is a principal idempotent of $S$. It is easy to show [5] that these two definitions are consistent. If $a$ is any element of $S$, then an inverse $a^{\prime}$ of $S$ will be called a principal inverse of $a$ if $a^{\prime} a$ is a principal idempotent of $S$. If $a \in S$, then $E_{a}$ will denote the set of the principal inverses of $a$. Following [1] and [5], a semigroup $S$ is called a weakly inverse semigroup if for every $a \in S, E_{a} \neq \square$, and for every $a, b \in S$ we have

(i) $E_{a b} \leqq E_{b} E_{a}$,

(ii) $E_{a}=E_{b}$ implies $a=b$.

The following lemma summarizes some of the results of [5].

LEMMA 2.1. If $S$ is a weakly inverse semigroup, then

(i) the principal idempotents of $S$ form a semilattice,

(ii) $E_{a} a$ consists of a single idempotent $e_{a}$ for every $a \in S$,

(iii) every principal left ideal of $S$ has a unique principal idempotent generator, 
(iv) the set $I$ of the principal elements of $S$ forms an inverse subsemigroup of $S$;

(v) an element $a \in S$ is a principal element of $S$ if and only if a has a unique principal inverse;

(vi) for every $a, b \in S$, we have $E_{a b}=E_{b}^{a} E_{a}$, where

$$
E_{b}^{a}=\left\{b^{\prime} \in E_{b}: e_{a} b b^{\prime} e_{a}=e_{a} b b^{\prime}\right\} \text {. }
$$

If $a$ is any element of the weakly inverse semigroup $S$, then $a^{\prime}, a_{1}^{\prime}, \cdots$ will denote principal inverses of $a$, whereas $a^{\prime \prime}$ will denote the unique principal inverse of $a^{\prime} \in E_{a}$.

The semigroup $T(X)$ of the partial transformations on the set $X$ is a weakly inverse semigroup. An element $\alpha \in T(X)$ is a principal element of $T(X)$ if and only if it is a one-to-one partial transformation on the set $X$. The Semigroup $T(X)$ will be called the symmetric weakly inverse semigroup on the set $X$ [5]. Let us recall the main theorem of [5]:

LEMмA 2.2. Let $S$ be a weakly inverse semigroup. For any $a \in S$ let $\psi_{a}$ be the partial transformation on $S$ where $\operatorname{dom} \psi_{a}=S E_{a}$, and where for every $x \in \operatorname{dom} \psi_{a}, x \psi_{a}=x a$. The mapping $S \rightarrow T(S)$, $a \rightarrow \psi_{a}$ embeds $S$ isomorphically into the symmetric weakly inverse semigroup $T(S)$ in such a way that an element $a \in S$ is principal in $S$ if and only if $\psi_{a}$ is principal in $T(S)$.

With the notation of Lemma 2.2 we now have the following

LEMMA 2.3. Let $S$ be a weakly inverse semigroup, and let a and $b$ be elements of $S$. The following conditions are equivalent:

(i) $E_{a} b=\left\{e_{a}\right\}$,

(ii) for every $a^{\prime} \in E_{a}$ there exists a $b^{\prime} \in E_{b}$ such that $a^{\prime} \leqq b^{\prime}$ in $I$,

(iii) $\psi_{a} \sqsubseteq \psi_{b}$.

Proof. (i) $\Longrightarrow$ (ii). Let $a^{\prime}$ be any element of $E_{a}$. By Lemma 2.1 (vi), there exists a $b^{\prime} \in E_{b}$ such that $b^{\prime} a^{\prime \prime} \in E_{a \prime b}$. Since $a^{\prime} b=e_{a}=a^{\prime} a^{\prime \prime}$ we have $b^{\prime} a^{\prime \prime}=a^{\prime} a^{\prime \prime}$, and so $a^{\prime} \leqq b^{\prime}$ in $I$.

(ii) $\Rightarrow$ (i). Ler $a^{\prime}$ be any element of $E_{a}$, and let $b^{\prime}$ be an element of $E_{b}$ such that $a^{\prime} \leqq b^{\prime}$ in $I$. Then $a^{\prime} b=a^{\prime} b^{\prime \prime} b^{\prime} b=a^{\prime} b^{\prime \prime}=a^{\prime} a^{\prime \prime}=e_{a}$. Therefore (i) holds.

(i) $\Leftrightarrow$ (ii) $\Longrightarrow$ (iii). Let $x$ be any element of dom $\psi_{a}$. Then there exists a $a^{\prime} \in E_{a}$ such that $x=x a^{\prime \prime} a^{\prime}$. Let $b^{\prime}$ be any element of $E_{b}$ such that $a^{\prime} \leqq b^{\prime}$ in $I$. Then $x=x a^{\prime \prime} a^{\prime}=x a^{\prime \prime} a^{\prime} b^{\prime \prime} b^{\prime}=x b^{\prime \prime} b^{\prime} \in \operatorname{dom} \psi_{b}$; moreover $x b=x b^{\prime \prime} b^{\prime} b=x b^{\prime \prime}=x a^{\prime \prime} a^{\prime} b^{\prime \prime}=x a^{\prime \prime} a^{\prime} a^{\prime \prime}=x a^{\prime \prime} a^{\prime} a=x a$. We conclude that $\psi_{a} \subseteq \psi_{b}$. 
(iii) $\Rightarrow$ (i) $\Leftrightarrow$ (ii). Let $a^{\prime}$ be any element of $E_{a}$. Since $E_{a} \subseteq$ dom $\psi_{a} \subseteq$ $\operatorname{dom} \psi_{b}$, we have $e_{a}=a^{\prime} a=a^{\prime} \psi_{a}=a^{\prime} \psi_{b}=a^{\prime} b$. Hence $E_{a} b=\left\{e_{a}\right\}$.

It follows from Lemma 2.2 and Lemma 2.3 that the relation $\leqq$ on the weakly inverse semigroup $S$ which is defined by $a \leqq b$ if and only if $a$ and $b$ satisfy the equivalent conditions of Lemma 2.3, must be a partial order on $S$ which is compatible with the multiplication. We shall call this partial order the natural partial order on the weakly inverse semigroup $S$. The natural partial order $\leqq$ induces the usual natural partial order on the inverse subsemigroup $I$. However, $\leqq$ does not induce the usual natural partial order on the idempotents of $S$ in the general case; indeed, if $f=f^{2}$ is an idempotent of $S$ which is not principal in $S$, then $f \neq e_{f}, f \mathscr{L} e_{f}$ and $e_{f} \leqq f$, whereas $e_{f}$ cannot be below $f$ for the usual natural partial order on the set of idempotents of $S$. The above defined natural partial order on the weakly inverse semigroup $S$. The above defined natural partial order on the weakly inverse semigroup $S$ will henceforth be denoted by $\leqq$.

Lemma 2.4. If $S$ is any weakly inverse semigroup, then $I$ is an order ideal of $S, \leqq$.

Proof. Let $b$ be any element of $I$, and suppose that $a \leqq b$ in $I$. Clearly $E_{b}=\left\{b^{\prime}\right\}$ is a singletion. If $a^{\prime}, a_{1}^{\prime}$ are any elements of $E_{a}$, then $a \leqq b$ implies that $a^{\prime} \leqq b^{\prime}$ and $a_{1}^{\prime} \leqq b^{\prime}$ in $I$. Since $a^{\prime} \mathscr{R} a_{1}^{\prime}$ in the inverse semigroup $I$, we must have $a^{\prime}=a_{1}^{\prime}$. Hence $E_{a}$ is a singleton, and by Lemma 2.1 (v) it follows that $a \in I$.

LEMMA 2.5. If $e$ is a principal idempotent of the weakly inverse semigroup $S$, and $a \in S$, then $e a \leqq a$ and $a e \leqq a$.

Proof. Any element of $E_{\theta a}$ is of the form $a^{\prime} e$ for some element $a^{\prime} \in E_{a}$ by Lemma 2.1(vi). Hence $\left(a^{\prime} e\right) a=\left(a^{\prime} e\right)(e a)=e_{e a}$. Thus $E_{e a}=$ $\left\{e_{e a}\right\}$, and so $e a \leqq a$.

Any element of $E_{a e}$ is of the form $e a^{\prime}$, where $a^{\prime} \in E_{a}$ by Lemma 2.1. (vi). Then $e a^{\prime} a=e a^{\prime} a e=e_{a e}$, thus $E_{a e} a=\left\{e_{a e}\right\}$, and so $a e \leqq a$.

LEMMA 2.6. Let $S$ be a weakly inverse subsemigroup of the symmetric weakly inverse semigroup $T(X)$ on the set $X$. Let us suppose that for every $\alpha \in S$ and for every $x \in \operatorname{dom} \alpha$ there exists a principal inverse $\alpha^{\prime}$ of $\alpha$ in $S$ such that $x \alpha \alpha^{\prime}=x$. Then the natural partial order on $S$ coincides with the inclusion relation for partial transformations. 
Proof. Let $\alpha$ and $\beta$ be any elements of $S$ such that $\alpha \leqq \beta$, and let us suppose that $x \in \operatorname{dom} \alpha$. There exists $\alpha^{\prime} \in E_{\alpha}$ such that $x \alpha \alpha^{\prime}=x$. From $\alpha \leqq \beta$ it follows that $\alpha^{\prime} \beta=\alpha^{\prime} \alpha$, and so $x \alpha=$ $x \alpha \alpha^{\prime} \alpha=x \alpha \alpha^{\prime} \beta=x \beta$. Hence $\alpha \subseteq \beta$. Let us conversely suppose that $\alpha$ and $\beta$ are elements of $S$ such that $\alpha \cong \beta$. Let $\alpha^{\prime}$ be any element of $E_{\alpha}$. Clearly $\operatorname{dom} \alpha^{\prime} \alpha=\operatorname{dom} \alpha^{\prime} \beta$. If $x \in \operatorname{dom} \alpha^{\prime} \beta$, then $x \in \operatorname{dom} \alpha^{\prime}=$ $\operatorname{dom} \alpha^{\prime} \alpha$, and so $\operatorname{dom} \alpha^{\prime} \alpha=\operatorname{dom} \alpha^{\prime} \beta$. From $\alpha \subseteq \beta$ it now follows that $\alpha^{\prime} \alpha=\alpha^{\prime} \beta$. Hence $E_{\alpha} \alpha=\left\{e_{\alpha}\right\}$, and we conclude that $\alpha \subseteq \beta$.

The following alternative characterization of weakly inverse semigroups will be used later in this paper.

THEOREM 2.7. For a regular semigroup $S$ the following conditions are equivalent:

(i) $S$ is a weakly inverse smigroup.

(ii) There exists a commutative subsemigroup $E$ of idempotents of $S$ such that

(a) for every $a \in S$ the set $C_{a}$ of inverses $a^{\prime}$ of a for which $a^{\prime} a \in E$ is nonempty,

(b) $C_{a b} \subseteq C_{b} C_{a}$ for all $a, b \in S$,

(c) $C_{a}=C_{b}$ implies $a=b$ for all $a, b \in S$.

Proof. That (i) implies (ii) is immediate. Let us now suppose that (ii) holds. Let $e$ be any element of $E$, let $f=f^{2}$ be any idempotent of $S$, and suppose that $f^{\prime} \in C_{f}$. Then

$$
f e f=f\left(f^{\prime} f\right) e f=f e\left(f^{\prime} f\right) f=f e\left(f^{\prime} f\right)=f\left(f^{\prime} f\right) e=f e,
$$

and so $e$ is a principal idempotent of $S$. Let $f=f^{2}$ be any principal idempotent of $S$, and suppose that $f^{\prime} \in C_{f}$. Then $f^{\prime} f$ is the idempotent which belongs to $E$, and which is $\mathscr{L}$-related to $f$. Using the fact that $f$ is principal we have

$$
f^{\prime}=f^{\prime}\left(f f^{\prime}\right)=f^{\prime}\left(f f^{\prime}\right) f\left(f f^{\prime}\right)=f^{\prime}\left(f f^{\prime}\right) f=f^{\prime} f .
$$

Thus $C_{f}$ is the singleton which consists of the element $f^{\prime} f=f^{\prime}$ which is $\mathscr{L}$-related to $f$; clearly $C_{f \prime}=\left\{f^{\prime}\right\}$ and so $C_{f}=C_{f^{\prime}}$. Hence $f=f^{\prime} \in E$. We conclude that $E$ is precisely the set of principal idempotents of $S$. Consequently, $S$ is a weakly inverse semigroup.

3. Right skew semigroups. A semigroup $R$ is called a right skew semigroup if for all $x, y, a \in R, x a=y a$ implies that there exists a left identity $e$ of $R$ such that $x=y e$.

If $a$ is any element of the right skew semigroup $R$, then $a^{2}=a^{2}$ implies that $a=a e$ for some left identity $e$ of $R$. This already indicates that the set of left identities of $R$ is nonempty. If $f$ is 
any idempotent of $R$, and $e$ any left identity of $R$, then $e f=f$ implies that there exists a left identity $g$ of $R$ such that $f=e g=g$. We conclude that the set of idempotents of $R$ coincides with the set of left identities of $R$. It is then obvious that the set of idempotents of $R$ forms a right zero semigroup.

We shall now provide an example of a right skew semigroup. Let $X$ be a set, and $\mu$ an equivalence relation of $X$. Let $\mathscr{T}_{\mu}(X)$ be the set of transformations of the set $X$ where

(i) $\operatorname{Ker} \alpha=\mu$,

(ii) $(x \alpha, y \alpha) \in \mu$ implies $(x, y) \in \mu$ for all $x, y \in X$.

In the terminology of [4] ${ }^{\prime \prime}(X)$ is the semigroup of all $\mu$-transformations with domain $X$.

THEOREM 3.1. T $\mathscr{S}^{\prime}(X)$ is a subsemigroup of the full transformation semigroup on the set $X$ which is a right skew semigroup. Every right skew semigroup $R$ can be represented faithfully by a semigroup of $\mu$-transformation with domain $R$.

Proof. It follows from [4] that $\mathscr{Y}_{\mu}(X)$ is a subsemigroup of the full transformation semigroup on the set $X$. Let us now suppose that $\varphi \alpha=\psi \alpha$ for some $\varphi, \psi, \alpha \in \mathscr{T}_{\mu}(X)$. Since $X_{\psi}$ intersects every $\mu$-class in at most one element we can choose an idempotent $\varepsilon \in \mathscr{S}_{\mu}(X)$ such that $X \psi \subseteq X \varepsilon$. From $\operatorname{Ker} \varepsilon=\operatorname{Ker} \alpha=\mu$ it follows that $\varepsilon$ and $\alpha$ are $\mathscr{R}$-related in the full transformation semigroup on the set $X$. Therefore $\varphi \alpha=\psi \alpha$ implies $\varphi \varepsilon=\psi \varepsilon$, where $\psi \varepsilon=\psi$ since $X \psi \subseteq X \varepsilon$. Obviously $\varepsilon$ is a left identity of $\mathscr{T}_{\mu}(X)$. We conclude that $\mathscr{T}_{\mu}(X)$ is a right skew semigroup.

If $R$ is a right skew semigroup, then

$$
\begin{aligned}
\mu & =\{(x, y) \in R \times R: x a=y a & \text { for some } & a \in R\} \\
& =\{(x, y) \in R \times R: x a=y a & \text { for all } & a \in R\}
\end{aligned}
$$

is a congruence relation on $R$, and the right regular representation of $R$ provides a representation of $R$ by a subsemigroup of $\mathscr{T}_{\mu}(R)$. Since $R$ contains left identities, the right regular representation of $R$ is faithful.

A right zero subsemigroup $E$ of a weakly inverse semigroup $S$ will be called a system of partial identities of $S$ if the following conditions are satisfied.

(i) If $a$ in any nonprincipal element of $S$, and $e \in E$, then $e a=a$.

(ii) If $f=f^{2}$ is any idempotent of $S$, then there exists an $e \in E$ such that $f \leqq e$.

We remark that $\leqq$ always denotes the natural partial order on 
the weakly inverse semigroup $S$, as defined in $\S 2$. If $S$ is an inverse semigroup, then $E$ must be a singleton. Conversely, if $E$ is a singleton, then $E=\{e\}$, and $E_{e}$ must be a singleton; by Lemma 2.1 (v) it then follows that $e$ is a principal idempotent; since $f \leqq e$ for every idempotent $f \in S$, we conclude that $f$ must be principal by virtue of Lemma 2.4; Hence $S$ is an inverse semigroup with identity $e$. Consequently, a weakly inverse semigroup $S$ with a system $E$ of partial identities is an inverse monoid if and only if $E$ is a singleton.

THEOREM 3.2. If $S$ is a weakly inverse semigroup with a system $E$ of partial identities, then the $\mathscr{R}$-class $R$ of $S$ which contains the partial identities is a right skew subsemigroup of $S$.

Proof. Let $a$ and $b$ be any elements of $R$, and let $e_{a}$ be the principal idempotent which is $\mathscr{L}$-related to $a$. There exists an $e \in E$ such that $e_{a} \leqq e$. This condition implies that $E_{e_{a}} e=\left\{e_{a}\right\}$ or, $e_{a} e=e_{a}$. Consequently $a e=a$. Since $e$ is $\mathscr{R}$-related to $b$, there exists a $b^{\prime} \in E_{b}$ such that $b b^{\prime}=e$. Then $a b b^{\prime}=a e=a$ implies that $a b$ is $\mathscr{R}$-related to $a$, hence $a b \in R$. We conclude that $R$ is a subsemigroup of $S$. Let $c$ be any other element of $R$, and suppose that $a c=b c$. Let $c^{\prime} \in E_{c}$, where $c c^{\prime}=e$. Then $a c=b c$ implies that $b e=a e=a$, where $e \in R$ is a left identity of $R$. Thus, $R$ is a right skew subsemigroup of $S$.

We now proceed to show the converse for Theorem 3.2. We shall show that, given any right skew semigroup $R$, we can construct a weakly inverse semigroup with a system of partial identities in such a way that the $\mathscr{R}$-class which contains the partial identities is a right skew semigroup which is isomorphic to $R$.

In the remainder of this section $R$ will denote a right skew semigroup, and $E$ the set of idempotents of $R$. We know from Theorem 3.1, that the right regular representation $\rho$ maps $R$ isomorphically into the symmetric weakly inverse semigroup $T(R)$. For any $\alpha \in T(R)$, let $E_{\alpha}$ denote the set of principal inverses of $\alpha$ in $T(R)$. Define

$$
(R \rho)^{\prime}=\left\{\alpha^{\prime} \in E_{\alpha}: \alpha \in R \rho \text { and } \alpha \alpha^{\prime} \in R \rho\right\},
$$

and let

$$
(R \rho)^{\prime \prime}=\left\{\alpha^{\prime \prime} \in E_{\alpha^{\prime}}: \alpha^{\prime} \in(R \rho)^{\prime}\right\} .
$$

Let $\sum$ be the subsemigroup of $T(R)$ which is generated by the elements of $R \rho \cup(R \rho)^{\prime} \cup(R \rho)^{\prime \prime}$. We shall show that the semigroup $\Sigma$ is a weakly inverse semigroup with a system of partial identities, and that $R \rho$ is the $\mathscr{R}$-class of $\sum$ which contains the partial identities. 
LEMMA 3.3. For every $\alpha \in R \rho$ and every $\varepsilon=\varepsilon^{2} \in R \rho$ there exists an $\alpha^{\prime} \in E_{\alpha} \cap(R \rho)^{\prime}$ such that $\alpha \alpha^{\prime}=\varepsilon \cdot R \rho$ is an $\mathscr{R}$-class of $\sum$.

Proof. Let $\alpha \in R \rho$, and $\varepsilon=\varepsilon^{2} \in R \rho$. Then $\alpha=a \rho$ and $\varepsilon=e \rho$ for some $a, e=e^{2} \in R$. The mapping $\alpha^{\prime}: R a \rightarrow R e, x a \rightarrow x e$ is a welldefined one-to-one partial transformation on the set $R$, and it is easy to see that $\alpha^{\prime} \in E_{\alpha} \cap(R \rho)^{\prime}$ and $\alpha \alpha^{\prime}=\varepsilon$. This already indicates that $R \rho$ is contained in an $\mathscr{R}$-Class of $\sum$.

If $\alpha \in R \rho$ then obviously $\operatorname{dom} \alpha=R$, and $\alpha$ is a right translation of $R$. Let $\alpha$ be any element of $R \rho$, and let $\alpha^{\prime} \in E_{\alpha}$, where $\alpha \alpha^{\prime} \in R \rho$. Let $s \in \operatorname{dom} \alpha$ and $s \alpha^{\prime}=q$. Since $\alpha^{\prime} \alpha$ is the restriction to $\operatorname{dom} \alpha^{\prime}$ of the identity mapping, we have $s \alpha^{\prime} \alpha=q \alpha=s$. For any $r \in R,(r q) \alpha=r(q \alpha)=r s$, and so $r s \in \operatorname{dom} \alpha^{\prime}$. Moreover, $(r s) \alpha^{\prime}=$ $(r q) \alpha \alpha^{\prime}=r\left(q \alpha \alpha^{\prime}\right)=r\left(s \alpha^{\prime}\right)$ and so we may conclude that, whenever $s \in \operatorname{dom} \alpha^{\prime}$, then $r s \in \operatorname{dom} \alpha^{\prime}$ for all $r \in R$, and $(r s) \alpha^{\prime}=r\left(s \alpha^{\prime}\right)$. In other words, $\alpha^{\prime}$ is a partial right translation for all $\alpha^{\prime} \in(R \rho)^{\prime}$. Let $\alpha^{\prime \prime} \in(R \rho)^{\prime \prime}$, where $\alpha^{\prime \prime} \in E_{\alpha^{\prime}}$, with $\alpha^{\prime} \in E_{\alpha}$ and $\alpha \alpha^{\prime} \in R \rho$. Since $\alpha^{\prime \prime} \alpha^{\prime} \in$ $E_{\alpha \alpha^{\prime}}$, where $\alpha \alpha^{\prime} \in R \rho$ and $\left(\alpha \alpha^{\prime}\right)\left(\alpha^{\prime \prime} \alpha^{\prime}\right) \in R \rho$ it follows that $\alpha^{\prime \prime} \alpha^{\prime} \in(R \rho)^{\prime}$ is a partial right translation of $R$. Thus $\alpha^{\prime \prime}=\left(\alpha^{\prime \prime} \alpha^{\prime}\right) \alpha$ being a composition of partial right translations of $R$ must also be a partial right translation of $R$. We showed that every element of $R \rho U$ $(R \rho)^{\prime} \cap(R \rho)^{\prime \prime}$ must be a partial right translation of $R$. Thus, all elements of $\Sigma$ are partial right translation of $R$. If $\xi$ is any element in the $\mathscr{R}$-class which contains $R \rho$ as a subset, then $\operatorname{dom} \xi=R$, and so $\xi$ must be a right translation of $R$. If $\xi$ is any fixed left identity of $R$, then $f \rho$ is an idempotent of $R \rho$, and there exists a $\xi^{\prime} \in \Sigma$ such that $\xi \xi^{\prime}=f \rho$. If $g$ is any left identity of $R$, then $g \xi=$ $g \xi \xi^{\prime} \xi=g f \xi=f \xi$. If $r$ is any element of $R$, then there exists a left identity $e$ of $R$ such that $r e=r$, and then $r \xi=(r e) \xi=r(e \xi)=r(f \xi)$. We conclude that $\xi=(f \xi) \rho \in R \rho$. Thus $R \rho$ is an $\mathscr{R}$-class of $\Sigma$.

Lemma 3.4. If $\alpha \in R \rho$ and $\beta^{\prime} \in(R \rho)^{\prime}$, then $\beta^{\prime} \alpha=\beta^{\prime} \alpha^{\prime \prime}$, where $\alpha^{\prime \prime} \in(R \rho)^{\prime \prime} \cap E_{\alpha^{\prime}}$ for some $\alpha^{\prime} \in(R \rho)^{\prime} \cap E_{\alpha}$ for which $\alpha \alpha^{\prime} \in R \rho$. If $\beta^{\prime \prime} \in$ $(R \rho)^{\prime \prime}$, then $\beta^{\prime \prime} \alpha=\beta^{\prime \prime} \alpha_{1}^{\prime \prime}$, where $\alpha_{1}^{\prime \prime} \in(R \rho)^{\prime \prime} \cap E_{\alpha_{1}^{\prime}}$ for some $\alpha_{1}^{\prime} \in(R \rho)^{\prime} \cap E_{\alpha}$ for which $\alpha \alpha_{1}^{\prime} \in R \rho$.

Proof. There exists a $\beta \in R \rho$ such that $\beta^{\prime} \in(R \rho)^{\prime} \cap E_{\beta}$ and $\left\{\beta^{\prime \prime}\right\}=E_{\beta^{\prime}}$. By Lemma 3.3 there exists $a \alpha^{\prime}$ in $E_{\alpha} \cap(R \rho)^{\prime}$ such that $\alpha \alpha^{\prime}=\beta \beta^{\prime}$. Let $\alpha^{\prime \prime}$ be the unique element of $E_{\alpha}$. Clearly $\alpha^{\prime \prime} \in(R \rho)^{\prime \prime}$. From $\alpha \alpha^{\prime}=\beta \beta^{\prime}$ it follows that $\beta^{\prime} \mathscr{L} \alpha^{\prime} \mathscr{L} \alpha^{\prime \prime} \alpha^{\prime}$, and so $\beta^{\prime} \alpha=\beta^{\prime} \alpha^{\prime \prime} \alpha^{\prime} \alpha^{\prime}=$ $\beta^{\prime} \alpha^{\prime \prime}$.

Since $R \rho$ is a right skew semigroup, there exists a left identity $\varepsilon$ of $R \rho$ such that $\beta=\beta \varepsilon$. By Lemma 3.3, there exists $a \alpha_{1}^{\prime}$ in $E_{\alpha} \cap$ $(R \rho)^{\prime}$ such that $\alpha \alpha_{1}^{\prime}=\varepsilon$. Let $\alpha_{1}^{\prime \prime}$ be the unique element of $E_{\alpha_{1}^{\prime}}$. 
Clearly $\alpha_{1}^{\prime \prime} \in(R \rho)^{\prime \prime}$ and $\beta^{\prime \prime} \alpha=\beta^{\prime \prime} \alpha \alpha_{1}^{\prime} \alpha=\beta^{\prime \prime} \alpha \alpha_{1}^{\prime} \alpha_{1}^{\prime \prime}=\beta^{\prime \prime} \varepsilon \alpha_{1}^{\prime \prime}=\beta^{\prime \prime} \beta^{\prime} \beta \varepsilon \alpha_{1}^{\prime \prime}=$ $\beta^{\prime \prime} \beta^{\prime} \beta \alpha_{1}^{\prime \prime}=\beta^{\prime \prime} \alpha_{1}^{\prime \prime}$.

LEMMA 3.5. Let $I$ be the subsemigroup of $\Sigma$ which is generated by the elements of $(R \rho)^{\prime} \cup(R \rho)^{\prime \prime}$. Then $I$ is an inverse subsemigroup of $\Sigma$, and all the elements of $I$ are principal in $\Sigma$. Moreover $\Sigma=$ $(R \rho) I \cup I$.

Proof. It is clear that $I$ consists of elements which are principal in $T(R)$, and so must be a subsemigroup of the symmetric inverse semigroup on the set $R$, i.e., the semigroup of all one-to-one partial transformations on the set $R$. Since $I$ is generated by a set of elements together with their inverses, $I$ must be an inverse subsemigroup of the symmetric inverse semigroup on the set $R$. Since all the idempotents of $I$ are principal in $T(R)$ we must have all the elements of $I$ are principal in $\Sigma$. That $\Sigma=(R \rho) I \cup I$ follows immediately from Lemma 3.4 .

Lemma 3.6. For any $\xi \in \Sigma$, let $G_{\xi}$ denote the set of inverses $\xi^{\prime}$ of $\xi$ in $\Sigma$ such that $\xi^{\prime} \xi \in I$. Then $G_{\xi}=E_{\xi} \cap \Sigma \neq \square$. For every $\alpha \in R \rho$ and every $\zeta \in I$ we have $G_{\alpha \zeta}=G_{\zeta} G_{\alpha}$.

Proof. If $\xi \in \Sigma$, then $\xi \in I$ or $\xi \in(R \rho) I$. If $\xi \in I$, then $G_{\xi}=E_{\xi}=$ $E_{\xi} \cap \Sigma$ is the singleton $\left\{\xi^{\prime}\right\}$ where $\xi^{\prime}$ is the unique inverse of $\xi$ in $I$. Let us now suppose that $\xi=\alpha \zeta$, where $\alpha \in R \rho$ and $\zeta \in I$. By Lemma $3.3 G_{\alpha} \neq \square$. If $\zeta^{\prime}$ is the unique inverse of $\zeta$ in $I$ and $\alpha^{\prime} \in G_{\alpha} \subseteq E_{\alpha} \cap \Sigma$, then $\zeta^{\prime} \alpha^{\prime}$ is an element of $I$ which is an inverse of $\alpha \zeta$, where $\zeta^{\prime} \alpha^{\prime} \alpha \zeta$ is an idempotent of $I$. Consequently $\square \neq G_{\zeta} G_{\alpha} \subseteq G_{\alpha \zeta} \subseteq E_{\alpha \zeta} \cap \Sigma$. Let us now suppose that $(\alpha \zeta)^{\prime}$ is any element of $E_{\alpha \zeta} \cap \Sigma$. Since $E_{\alpha \zeta} \subseteq$ $E_{\zeta} E_{\alpha}=\zeta^{\prime} E_{\alpha}$, where $\zeta^{\prime}$ is the unique inverse of $\zeta$ in $I$, we must have that $(\alpha \zeta)^{\prime}$ is of the form $\zeta^{\prime} \alpha_{1}^{\prime}$ for some $\alpha_{1}^{\prime} \in E_{\alpha}$. Obviously $(\alpha \zeta)\left(\zeta^{\prime} \alpha_{1}^{\prime}\right) \in \Sigma$, and so $\alpha \zeta \zeta^{\prime} \alpha_{1}^{\prime}=\beta_{1} \cdots \beta_{n}$, where $\beta_{i} \in R \rho \cup(R \rho)^{\prime} \cup(R \rho)^{\prime \prime}$ for all $i=1, \cdots, n$. Since $\alpha \zeta \zeta^{\prime} \alpha^{\prime} \in(R \rho) I \cup I$ we may suppose that $\beta_{n} \in(R \rho)^{\prime}$ or $\beta_{n} \in(R \rho)^{\prime \prime}$. In both cases $\beta_{n} \mathscr{L} \beta$ for some $\beta \in R \rho$. There exists a left identity $\varepsilon$ of $R \rho$ such that $\beta \varepsilon=\beta$, and then $\alpha \zeta \zeta^{\prime} \alpha_{1}^{\prime} \varepsilon=\alpha \zeta \zeta^{\prime} \alpha_{1}^{\prime}$. Let $\alpha_{2}^{\prime}$ be any element of $(R \rho)^{\prime} \cap E_{\alpha}$ such that $\alpha \alpha_{2}^{\prime}=\varepsilon$. Clearly $\alpha_{2}^{\prime} \in G_{\alpha}$ and $\alpha \zeta \zeta^{\prime} \alpha_{1}^{\prime}=\alpha \zeta \zeta^{\prime} \alpha_{1}^{\prime} \varepsilon=\alpha \zeta \zeta^{\prime} \alpha_{1}^{\prime} \alpha \alpha_{2}^{\prime}=\alpha \zeta \zeta^{\prime} \alpha_{2}^{\prime}$. Since also $\zeta^{\prime} \alpha_{2}^{\prime} \in E_{\alpha \zeta}$ : we have $\zeta^{\prime} \alpha_{1}^{\prime} \alpha \zeta=\zeta^{\prime} \alpha_{2}^{\prime} \alpha \zeta$, and we conclude that $(\alpha \zeta)^{\prime}=\zeta^{\prime} \alpha_{1}^{\prime}=\zeta^{\prime} \alpha_{2}^{\prime} \in G_{\zeta} G_{\alpha}$. Thus $\square \neq G_{\zeta} G_{\alpha}=G_{\alpha \zeta}=E_{\alpha \zeta} \cap \Sigma$.

LEMMA 3.7. $\Sigma$ is a weakly inverse semigroup.

Proof. Let $\xi, \eta$ be any elements of $\Sigma$. If $\xi$, $\eta \in I$, then it is clear that $G_{\xi \mu}=G_{\mu} G_{\xi}$. If $\xi, \eta \in(R \rho) I$, then $\xi=\alpha \zeta$ and $\eta=\beta \theta$ for 
some $\alpha, \beta \in R \rho$ and $\zeta, \theta \in I$ by Lemma 3.4 , there exists a $\beta^{\prime \prime} \in I$, with $G_{\beta^{\prime \prime}} \subseteq G_{\beta}$, such that $\zeta \beta=\zeta \beta^{\prime \prime}$, and so

$$
\begin{aligned}
G_{\xi \eta}=G_{\alpha \zeta \beta \theta} & =G_{\alpha \zeta \beta^{\prime \prime} \theta}=G_{\zeta \beta^{\prime \prime} \theta} G_{\alpha}=G_{\beta^{\prime \prime \theta}} G_{\zeta} G_{\alpha} \\
& =G_{\theta} G_{\beta^{\prime \prime}} G_{\alpha \zeta} \subseteq G_{\theta} G_{\beta} G_{\alpha \zeta}=G_{\beta \theta} G_{\alpha \zeta}=G_{\eta} G_{\xi},
\end{aligned}
$$

by Lemma 3.6. The two other cases may be dealt with in a similar way, hence it follows from $\Sigma=(R \rho) I \cup I$ that $G_{\xi \eta} \subseteq G_{\eta} G_{\xi}$ for all $\xi, \mu \in \Sigma$.

Let $\xi=\alpha \zeta, \alpha \in R \rho, \zeta \in I$, be any element of $(R \rho) I$, and let us suppose that $G_{\xi}$ is a singleton. If $x \alpha \zeta=y \alpha \zeta$ for some $x, y \in R$, then $x \alpha=y \alpha$ since $\zeta$ is a one-to-one partial transformation. Putting $\alpha=a \rho$, we then have $x a=y a$, and since $R$ is right skew this implies $x=y e$ for some left identity $e$ of $R$. If $\varepsilon=e \rho$, then Lemma 3.3 guarantees that there exists a $\alpha^{\prime} \in G_{\alpha}$ such that $\alpha \alpha^{\prime}=\varepsilon$. If $\zeta^{\prime}$ is the unique element of $G_{\zeta}$, then $\zeta^{\prime} \alpha^{\prime} \in G_{\alpha \zeta}$. If $u=y \alpha \zeta \zeta^{\prime} \alpha^{\prime}$, then $u \alpha \zeta \zeta^{\prime} \alpha^{\prime}=y \alpha \zeta \zeta^{\prime} \alpha^{\prime}$, hence $u \alpha \zeta=y \alpha \zeta$. Again we may conclude that $y=u \lambda$ for some left identity $\lambda$ of $R \rho$, and that there exists a $\alpha_{1}^{\prime} \in G_{\alpha}$ such that $\alpha \alpha_{1}^{\prime}=\lambda$. Since both $\zeta^{\prime} \alpha^{\prime}$ and $\zeta^{\prime} \alpha_{1}^{\prime}$ belong to $G_{\alpha \zeta}$, and since $G_{\alpha_{5}}$ is a singleton, we must have $\zeta^{\prime} \alpha^{\prime}=\zeta^{\prime} \alpha_{1}^{\prime}$. Therefore

$$
y=u \lambda=y \alpha \zeta \zeta^{\prime} \alpha^{\prime} \lambda=y \alpha \zeta \zeta^{\prime} \alpha_{1}^{\prime} \alpha \alpha_{1}^{\prime}=y \alpha \zeta \zeta^{\prime} \alpha_{1}^{\prime}=y \alpha \zeta \zeta^{\prime} \alpha^{\prime}=u
$$

and so

$$
u=u \alpha \alpha^{\prime}=u e=y e=x,
$$

from which we have that $x=y$. Thus $\xi=\alpha \zeta$ is a one-to-one partial transformation on $R$, which implies that $\xi$ is a principal element of $T(R)$.

If $\xi$ and $\eta$ are any elements of $\Sigma$ such that $G_{\xi}=G_{\eta}$, and if $\eta \in I$, then $G_{\xi}=G_{\eta}=E_{\eta}$ is a singleton. By the foregoing this implies that $\zeta$ must be principal in $T(R)$, hence $G_{\xi}=E_{\xi}$. Since $T(R)$ is a weakly inverse semigroup $E_{\xi}=E_{\eta}$ then implies that $\xi=\eta$.

Let us now suppose that $\xi=\alpha \zeta$ and $\eta=\beta \theta$, where $\alpha, \beta \in R \rho$ and $\zeta, \theta \in I$, and $G_{\xi}=G_{\eta}$. Every element of $G_{\xi}$ is of the form $\zeta^{\prime} \alpha^{\prime}=\xi^{\prime}$ with $\zeta^{\prime} \in G_{\zeta}, \alpha^{\prime} \in G_{\alpha}$. Then $\xi^{\prime} \in G_{\eta}$, and so $\xi^{\prime} \eta=\xi^{\prime} \xi$. Since $\alpha \alpha^{\prime}$ is a left identity for $R \rho$ we also have $\alpha \alpha^{\prime} \eta=\alpha \alpha^{\prime} \beta \theta=\beta \theta=\eta$. Since $\zeta^{\prime} \zeta$ is the restriction of the identity transformation to dom $\zeta^{\prime} \zeta$ we have $\xi \xi^{\prime}=\alpha \zeta \zeta^{\prime} \alpha^{\prime} \subseteq \alpha \alpha^{\prime}$. Therefore

$$
\xi=\xi \xi^{\prime} \xi=\xi \xi^{\prime} \eta \cong \alpha \alpha^{\prime} \eta=\eta .
$$

One can show dually that $\eta \cong \xi$, and thus $\xi=\eta$. Since $\Sigma=(R \rho)$ $I \cup I$ we may conclude that $G_{\xi}=G_{\eta}$ implies $\xi=\eta$ for all $\xi, \eta \in \Sigma$. 
By Theorem 2.7 and Lemma 3.6 we have that $\Sigma$ is a weakly inverse semigroup.

We shall call $\Sigma$ the weakly inverse hull of the right skew semigroup $R$.

LEMMA 3.8. The set of idempotents of the $\mathscr{R}$-class $R \rho$ of form a system of partial identities of $\Sigma$.

Proof. Let $\xi=\alpha \zeta, \alpha \in R \rho, \zeta \in I$, be any element of $(R \rho) I$, and let $x \in \operatorname{dom} \alpha \zeta$. If $e$ is any left identity of $R$ such that $x=x e$, then there exists a $\alpha^{\prime} \in G_{\alpha}$ such that $\alpha \alpha^{\prime}=\varepsilon=e \rho$. If $\zeta^{\prime}$ is the unique element of $G_{\xi}$, then $\zeta^{\prime} \alpha^{\prime} \in G_{\xi}$ and $x \alpha \zeta \zeta^{\prime} \alpha^{\prime}=x \alpha \alpha^{\prime}=x \varepsilon^{\prime}=x e=x$. Hence for every $\xi \in(R \rho) I$ and every $x \in$ dom $\xi$ there exists a principal inverse $\xi^{\prime}$ of $\xi$ in $\Sigma$ such that $x \xi \xi^{\prime}=x$. Clearly if $\xi \in I$, and $x \in \operatorname{dom} \xi$, then also $x=x \xi \xi^{\prime}$ where $\xi^{\prime}$ is the unique element of $G_{\zeta}$. Since $\Sigma=(R \rho) I \cup I$ we conclude from Lemma 2.6 that the natural partial order on $\Sigma$ coincides with the inclusion relation for partial transformations.

Since every idempotent of the $\mathscr{R}$-class $R \rho$ is a left identity for $R \rho$, it must also be a left identity for the elements of the set $(R \rho)$ $I$ which contains all the nonprincipal elements of $\Sigma$.

Every idempotent of $\Sigma$ is of the form $\xi \xi^{\prime}$ where $\xi \in(R \rho) I$ or $\xi \in I$ and $\xi^{\prime} \in G_{\xi}$. If $\xi=\alpha \zeta$ where $\alpha \in R \rho$ and $\zeta \in I$, then $\xi^{\prime}$ is of the form $\zeta^{\prime} \alpha^{\prime}$ where $\alpha^{\prime} \in G_{\alpha}$ and $\zeta^{\prime} \in G_{\zeta}$. Clearly

$$
\xi \xi^{\prime}=\alpha \zeta \zeta^{\prime} \alpha^{\prime} \subseteq \alpha \alpha^{\prime} \in R \rho
$$

in this case, and so $\xi \xi^{\prime} \leqq \alpha \alpha^{\prime} \in R \rho$. Let us now suppose that $\xi \in I$. Then $\xi \xi^{\prime} \in I$, and $\xi \xi^{\prime}$ is of the form $\xi \xi^{\prime}=\beta_{1}, \cdots, \beta_{n}$, where $\beta_{i} \in$ $(R \rho)^{\prime} \cup(R \rho)^{\prime \prime}, i=1, \cdots, n$. In all cases $\beta_{n} \mathscr{L} \beta$ for some $\beta \in R \rho$. Since $R \rho$ is a right skew semigroup there exists an idempotent $\varepsilon$ in $R \rho$ such that $\beta \varepsilon=\beta$. Then $\xi \xi^{\prime} \varepsilon=\xi \xi^{\prime}$. Since $\xi \xi^{\prime} \in I$ is the restriction of the identity transformation to dom $\xi \xi^{\prime}$, we must have $\xi \xi^{\prime}=\xi \xi^{\prime} \varepsilon \subseteq \varepsilon$, and so $\xi \xi^{\prime} \leqq \varepsilon \in R \rho$.

We conclude that the set of idempotents of $R \rho$ forms a system of partial identities for $\Sigma$.

We summarize the results of Lemmas $3.3,3.4,3.5,3.6,3.7$ and 3.8 in the following theorem.

THEOREM 3.9. Let $R$ be any right skew semigroup and let $\Sigma$ be the weakly inverse hull of $R$. Then $\Sigma$ is a weakly inverse semigroup which contains $R$ as a subsemigroup and as an $\mathscr{R}$-class, and the set of idempotents of $R$ forms a system of partial identities for $\Sigma$. 
4. Bisimple weakly inverse semigroups with partial identities. In this section we characterize the right skew semigroups whose weakly inverse hull is a bisimple weakly inverse semigroup.

THEOREM 4.1. Let $S$ be a bisimple weakly inverse semigroup with a system of partial identities. Then the $\mathscr{R}$-class $R$ of $S$ which contains the partial identities is a right skew subsemigroup of $S$, where for every $a, b \in R$ there exists $a c \in R$ such that $R b \cap R b=$ Re.

Proof. It follows from Theorem 3.2 that $R$ is a right skew subsemigroup of $S$. Let $a, b \in R$, and let $\left\{e_{a}\right\}=E_{a} a,\left\{e_{b}\right\}=E_{b} b$. The principal idempotents form a commutative subsemigroup of $S$, and so $S a \cap S b=S e_{a} \cap S e_{b}=S e_{a} e_{b}$. Since $R$ is an $\mathscr{R}$-class of the bisimple semigroup $S$, there exists a $c \in R$ such that $S e_{a} e_{b}=S c$ and thus $S a \cap$ $S b=S c$ for some $c \in R$.

Let $x \in R \cap S a$. Then $x=s a$ for some $s \in S$. Since $S$ is bisimple there exists a $t \in L_{s} \cap R$, and since $R$ is a right skew semigroup, there exists an idempotent $e$ of $R$ such that $t e=t$. Then $s e=s$, with $e=e^{2} \in R$. Let $a^{\prime}$ be any inverse of $a$ in $S$ such that $a a^{\prime}=e$. Then $x=s a$ and $x a^{\prime}=s a a^{\prime}=s e=s$ imply that $s \in R$. Thus $x \in R a$, and so $S a \cap R \subseteq R a$. From this follows that $S a \cap R=R a$. Similarly $S b \cap R=R b$ and $S c \cap R=R c$. Hence from $S a \cap S b=S c$ we have $R a \cap R b=R c$.

THEOREM 4.2. Let $R$ be a right skew semigroup such that for every $a, b \in R, R a \cap R b=R c$ for some $c \in R$, and let $\Sigma$ be the weakly inverse hull of $R$. Then $\Sigma$ is a bisimple weakly inverse semigroup which contains $R$ as a subsemigroup and as an $\mathscr{R}$-Class, and the set of idempotents of $R$ forms a system of partial identities for $\Sigma$.

Proof. From Theorem 3.9, it follows that we only need to show that $\Sigma$ is a bisimple semigroup.

Let $\alpha$ and $\beta$ be any elements of $R \rho$, and let $\beta^{\prime} \in\left(R_{\rho} \rho\right)^{\prime} \cap E_{\beta}$. Let $\gamma$ be an element of $R_{\rho} \rho$ such that $(R \rho) \alpha \cap(R \rho) \beta=(R \rho) \gamma$. Putting $G_{\alpha} \alpha=\left\{e_{\alpha}\right\}, \quad G_{\beta} \beta=\left\{e_{\beta}\right\}$ and $G_{i} \gamma=\left\{e_{i}\right\}$ the foregoing implies that $e_{\alpha} e_{\beta}=e_{\gamma}$ since then $e_{\gamma}\left[\right.$ resp. $\left.e_{\alpha}, e_{\beta}\right]$ is the identity mapping on $R \gamma=$ $R \alpha \cap R \beta$ [resp. $R \alpha^{\prime}, R \beta$ ]. If $\left(\alpha \beta^{\prime} \beta\right)^{\prime}$ is any element of $G_{\alpha \beta^{\prime} \beta}=\beta^{\prime} \beta G_{\alpha}$, then $\left(\alpha \beta^{\prime} \beta\right)^{\prime} \alpha \beta^{\prime} \beta=e_{\beta} e_{\alpha} e_{\beta}=e_{i}$. Therefore $\alpha \beta^{\prime} \mathscr{R} \alpha \beta^{\prime} \beta \mathscr{L}^{\prime} \gamma$, and so $\alpha \beta^{\prime}$ belongs to the $\mathscr{D}$-class which contains $R \rho$ as an $\mathscr{R}$-class. Let $\alpha^{\prime}$ be any element of $(R \rho)^{\prime} \cap E_{\alpha}$ such that $\alpha \alpha^{\prime}=\beta \beta^{\prime}$; then $\beta^{\prime} \alpha^{\prime}$ and $\beta^{\prime} \alpha \mathscr{C} \alpha^{\prime} \alpha \alpha$, and so $\beta^{\prime} \alpha$ belongs to the $\mathscr{D}$-class which contains $R \rho$. If $\alpha_{1}^{\prime}$ is any element of $(R \rho)^{\prime} \cap E_{\alpha}$, then $\alpha_{1}^{\prime} \beta^{\prime} P\left(\alpha \alpha_{1}^{\prime}\right) \beta^{\prime}$, where $\alpha \alpha_{1}^{\prime} \in R \rho$, and by the foregoing we can again conclude that $\alpha_{1}^{\prime} \beta^{\prime}$ 
belongs to the $\mathscr{D}$-class which contains $R \rho$. We showed that the products of any two elements of $R \rho \cup(R \rho)^{\prime}$ belongs to the $\mathscr{D}$-class which contains $R \rho$. Let $\xi$ be any element of this $\mathscr{D}$-class, and let $\zeta$ be any element of $R \rho \cup(R \rho)^{\prime}$. If $\gamma$ is an element of $L_{\xi} \cap R \rho$, then $\gamma \zeta$ belongs to the $\mathscr{D}$-class which contains $R \rho$. Since $\xi \zeta \mathscr{L} \gamma \zeta$ this implies that also $\xi \zeta$ belongs to this $\mathscr{D}$-class. By induction we can then easily show that the subsemigroup of $\Sigma$ which is generated by the elements of $R \rho \cup(R \rho)^{\prime}$ is contained in this $\mathscr{D}$-class. If $\alpha \in R \rho$, $\alpha^{\prime} \in(R \rho)^{\prime} \cap E_{\alpha}$ and $\left\{\alpha^{\prime \prime}\right\}=E_{\alpha^{\prime}}$, then $\alpha^{\prime \prime}=\alpha^{\prime \prime} \alpha^{\prime} \alpha$, where $\alpha^{\prime \prime} \alpha^{\prime} \in E_{\alpha \alpha^{\prime}}$, and so $\alpha^{\prime \prime}$ is a product of elements of $R \rho \cup(R \rho)^{\prime}$. Hence $\Sigma$ is generated by the elements of $R \rho \cup(R \rho)^{\prime}$, and so $\Sigma$ is bisimple.

EXAMPLE. Let $A$ be a right concellative semigroup with an identity $e$, and let us suppose that $\leqq$ is a total order on the set $A$ where for any $a \in A, A a=\{x \in A a \leqq x\}$. Let $B$ be a semigroup which is isomorphic to $A$, and let $\rho: A \rightarrow B$ be an isomorphism of $A$ onto $B$. We shall suppose that there exists a $k \in A$ such that $x \varphi=x$ for all $x \in A k$, and that $A \cap B=A k$. On $R=A \cup B$ we define a multiplication which extends the separations on $A$ and on $B$ by

$$
\begin{aligned}
a b & =(a \varphi) b \quad \text { if } \quad a \in A \text { and } b \in B \\
& =\left(a \varphi^{-1}\right) b \text { if } a \in B \text { and } b \in A .
\end{aligned}
$$

It is easy to check that $R$ is a right skew semigroup which satisfies the conditions of Theorem 4.2.

ACKNowledgments. The author wishes to express his sincere thanks to Professor Francis Pastijn for his constructive and creative criticism that have led to considerable improvement of the paper, and his invaluable suggestions regarding style, diction and presentation of the paper. The author also thanks Dr. B. R. Srinivasan for his valuable suggestion and his work on the definition of natural partial order of weakly inverse semigroups.

\section{REFERENCES}

1. R. J. Brown, D. W. Hardy and R. J. Painter, A note on a paper by Srinivasan, Math. Ann., 186 (1970), 34-35.

2. A. H. Clifford and G. B. Preston, The Algebraic Theory of Semigrous, Vol. I, Providence, 1961, Vol. II, Providence, 1967.

3. S. Madhavan, On right normal right inverse semigroups, Semigroup Forum, 12 (1976), 333-339.

4. N. R. Reilly, Bisimple inverse semigroups, Trans. Amer. Math. Soc., 132 (1968), 101-114.

5. B. R. Srinivasan, Weakly inverse semigroups, Math. Ann., 176 (1968), 324-333. 
Received April 8, 1977 and in revised form August 29, 1979.

\section{UNIVERSITY COLLEGE}

TRIVANDRUM-695 001,

Kerala State, S. India 



\section{PACIFIC JOURNAL OF MATHEMATICS}

\section{EDITORS}

DONALD BABBITT (Managing Editor)

University of California

Los Angeles, CA 90024

Hugo RossI

University of Utah

Salt Lake City, UT 84112

C. C. MOORE and ANDREW OGG

University of California

Berkeley, CA 94720

\section{J. DugunduI}

Department of Mathematics

University of Southern California

Los Angeles, CA 90007

R. Finn and J. Milgram

Stanford University

Stanford, CA 94305

\section{ASSOCIATE EDITORS}
E. F. BeCKenBACH
B. H. NeumanN
F. WOLF
K. YosHIDA

\section{SUPPORTING INSTITUTIONS}

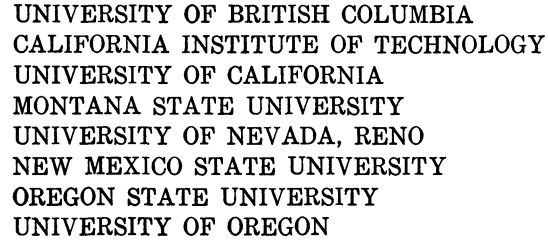

UNIVERSITY OF BRITISH COLUMBIA CALIFORNIA INSTITUTE OF TECHNOLOGY UNIVERSITY OF CALIFORNIA

MONTANA STATE UNIVERSITY

UNIVERSITY OF NEVADA, RENO

NEW MEXICO STATE UNIVERSITY

OREGON STATE UNIVERSITY UNIVERSITY OF OREGON

\author{
UNIVERSITY OF SOUTHERN CALIFORNIA \\ STANFORD UNIVERSITY \\ UNIVERSITY OF HAWAII \\ UNIVERSITY OF TOKYO \\ UNIVERSITY OF UTAH \\ WASHINGTON STATE UNIVERSITY \\ UNIVERSITY OF WASHINGTON
}

The Supporting Institutions listed above contribute to the cost of publication of this Journal, but they are not owners or publishers and have no responsibility for its content or policies.

Mathematical papers intended for publication in the Pacific Journal of Mathematics should be in typed form or offset-reproduced, (not dittoed), double spaced with large margins. Please do not use built up fractions in the text of the manuscript. However, you may use them in the displayed equations. Underline Greek letters in red, German in green, and script in blue. The first paragraph or two must be capable of being used separately as a synopsis of the entire paper. Please propose a heading for the odd numbered pages of less than 35 characters. Manuscripts, in triplicate, may be sent to any one of the editors. Please classify according to the scheme of Math. Reviews, Index to Vol. 39. Supply name and address of author to whom proofs should be sent. All other communications should be addressed to the managing editor, or Elaine Barth, University of California, Los Angeles, California, 90024.

50 reprints to each author are provided free for each article, only if page charges have been substantially paid. Additional copies may be obtained at cost in multiples of 50 .

The Pacific Journal of Mathematics is issued monthly as of January 1966. Regular subscription rate: $\$ 84.00$ a year (6 Vols., 12 issues). Special rato: $\$ 42.00$ a year to individual members of supporting institutions.

Subscriptions, orders for numbers issued in the last three calendar years, and changes of address shoud be sent to Pacific Journal of Mathematics, P.O. Box 969, Carmel Valley, CA 93924, U.S.A Old back numbers obtainable from Kraus Periodicals Co., Route 100, Millwood, NY 10546.

PUBLISHED BY PACIFIC JOURNAL OF MATHEMATICS, A NON-PROFIT CORPORATION

Printed at Kokusai Bunken Insatsusha (International Academic Printing Co., Ltd.). 8-8, 3-chome, Takadanobaba, Shinjuku-ku, Tokyo 160, Japan.

Copyright (C) 1980 by Pacific Jounal of Mathematics Manufactured and first issued in Japan 


\section{Pacific Journal of Mathematics \\ Vol. 90, No. $2 \quad$ October, 1980}

Frank Hayne Beatrous, Jr., Hölder estimates for the $\bar{\partial}$ equation with a support condition ..................................... 249

Charles L. Belna, Michael Jon Evans and Paul Humke, Planar continua

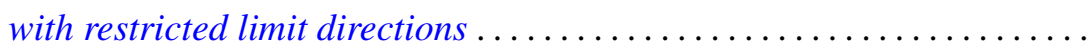

Leon Brown and Takashi Ito, Classes of Banach spaces with unique isometric preduals................................. 261

V. K. Deshpande, Completions of Noetherian hereditary prime rings ..... . 285

Deepak Dhar, Asymptotic enumeration of partially ordered sets . . . . . . . 299

Zeev Ditzian, On interpolation of $L_{p}[a, b]$ and weighted Sobolev

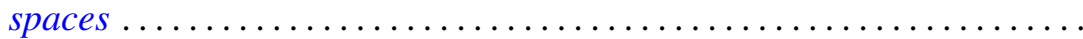

Andrew George Earnest, Congruence conditions on integers represented by

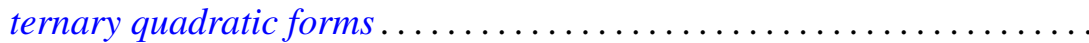

Melvin Faierman, Bounds for the eigenfunctions of a two-parameter system of ordinary differential equations of the second order ..............

Hector O. Fattorini, Vector-valued distributions having a smooth

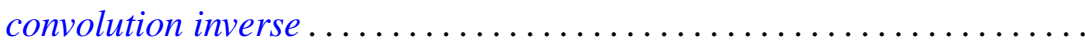

Howard D. Fegan, The spectrum of the Laplacian on forms over a Lie

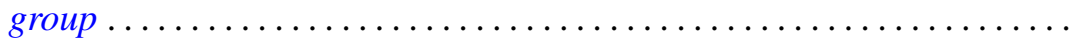

Gerald Leonard Gordon, On the degeneracy of a spectral sequence associated to normal crossings..

S. Madhavan, On bisimple weakly inverse semigroups ... 397

Françoise Mathot, On the decomposition of states of some

Roger McCann, Embedding asymptotically stable dynamical systems into radial flows in $l_{2}$

Michael L. Mihalik, Ends of fundamental groups in shape and proper homotopy...............................

Samuel Murray Rankin, III, Boundary value problems for partial functional differential equations .

Randy Tuler, Arithmetic sums that determine linear characters on $\Gamma(N)$

Jeffrey D. Vaaler, On linear forms and Diophantine approximation ..

G. P. Wene, Alternative rings whose symmetric elements are nilpotent or a right multiple is a symmetric idempotent. . 